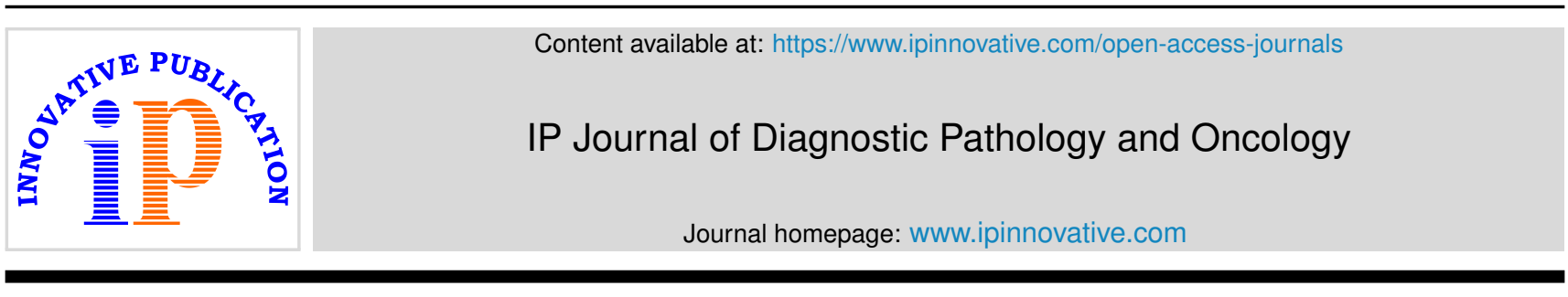

Original Research Article

\title{
A clinico-histopathological spectrum of Prostatic lesions at a tertiary care teaching institute
}

\author{
Tadepalli Krishna ${ }^{1, *}$ \\ ${ }^{1}$ Dept. of Pathology, SVS Medical College, Mahabubnagar, Telangana, India
}

\section{A R T I C L E I N F O}

\section{Article history:}

Received 01-08-2020

Accepted 11-08-2020

Available online 03-09-2020

\section{Keywords:}

Nodular hyperplasia

Basal cell hyperplasia

Adenocarcinoma prostate

Gleason's score

\begin{abstract}
A B S T R A C T
Background: Prostatic carcinoma is the second most common cause of cancer deaths in men and the incidence is increasing significantly in recent years, due to early screening measures. A study designed to identify the prostatic lesions spectrum as per histopathological classification.

Materials and Methods: 50 prostatic lesion samples collected in $10 \%$ formalin were subjected to $\mathrm{H}$ and $\mathrm{E}$ stain and the mean AgNOR counts were calculated.

Results: Out of 50 specimens, $80 \%$ were benign and $20 \%$ belongs to malignancy. The mean age of patient's benign and malignant lesions is $63.5 \pm 9.2$ years and $69.5 \pm 8.5$ years respectively $(\mathrm{P}=0.09)$. The most common clinical symptom of benign lesions was frequency in 35 patients, followed by hesitancy and nocturia in 33 and 30 patients respectively. Serum PSA values above $10 \mathrm{ng} / \mathrm{ml}$ were seen in 10 benign cases and 5 malignant cases $(\mathrm{P}=0.02)$. Out of 50 cases, 35 were $\mathrm{BPH}, 5$ were non-specific granulomatous prostatitis, 8 prostatic adenocarcinoma, 1 case urothelial carcinoma and 1case with both prostatic adenocarcinoma and urothelial carcinoma were recorded. High grade PIN identified in 9 specimens and low grade PIN identified in 8 specimens. High grade PIN shows association with adenocarcinoma. Basal cell hyperplasia identified in 3 specimens. Tufting Pattern was observed in 2 specimens, flat Pattern observed in 4 specimens, and cribriform pattern observed in 1 specimen out of 9. Majority of patients diagnosed as conventional adenocarcinoma with a score of 9 ( 3 cases) followed by score of 8 ( 2 cases).

Conclusion: BHP is the most common non neoplastic prostate lesion, and commonly encountered in age group of 60- 70 years. In the current scenario, histopathological analysis of prostate specimens plays crucial role in both diagnosis and management.
\end{abstract}

(C) 2020 Published by Innovative Publication. This is an open access article under the CC BY-NC license (https://creativecommons.org/licenses/by-nc/4.0/)

\section{Introduction}

Patients presenting with prostatic symptoms account for $22 \%$ of the total urology cases, and every $7^{\text {th }}$ patient will be presenting with urologic emergency. ${ }^{1}$

Prostatic carcinoma, the leading cause of cancer in western population after lung cancer which is leading cause of deaths in men. ${ }^{2}$ Almost $75 \%$ of the men diagnosed with prostatic carcinoma are aged 65 or older, but the tumours can be seen even in children and adolescents though, very rarely. ${ }^{3,4}$

\footnotetext{
* Corresponding author.

E-mail address: dr_k_tadepalli@yahoo.co.in (T. Krishna).
}

Prostatitis, benign nodular hyperplasia, and prostatic adenocarcinoma are the common diseases affecting men commonly after their fifth decade. ${ }^{5}$

The diagnosis of prostatic carcinoma is usually too late because there are no signs and symptoms specific to malignancy other than those for BPH. Advances in the field of biochemistry that have brought out PSA and other markers are valuable diagnostic tools, advances in the field of radiodiagnosis that have provided Transrectal ultrasound, advances in the field of instrumentation and biopsy procedure, TURP and prostatectomy that have led to the minimally invasive procedures like Needle biopsy, ongoing research in prostate pathology that led to the documentation of premalignant lesions like PIN \& AAH 
that predate malignancy by at least 10 years.

Current study tries to identify the proportion of the premalignant and malignant pattern in prostatic specimen received at our institution.

\section{Materials and Methods}

Current prospective study was carried out at the Department of Pathology, S.V.S. Medical College, Mahbubnagar, during June 2018 to May 2019. This study was conducted on 50 prostatic specimens referred to department of pathology. Brief clinical data were noted from the case records, which included the age, presenting symptoms, serum PSA levels and clinical diagnosis.

Following inclusion and exclusion criteria are adopted in this study.

\subsection{Inclusion criteria}

All types of prostatic specimens including TURP and prostatectomy are considered in this study.

\subsection{Exclusion criteria}

Inadequate biopsies and poorly preserved prostatic specimens are excluded.

PSA values, transrectal ultrasound findings and details for evidence of metastasis were taken whenever possible. All the prostatic specimens were subjected to a careful and detailed gross examination. $10 \%$ formalin fixed tissue sections from these specimens were used for microscopic study. 4-6 $\mu$ thick sections being prepared and stained routinely with $\mathrm{H} \& \mathrm{E}$. $\mathrm{H} \& \mathrm{E}$ stains were studied and classified into various benign and malignant lesions.

Different types of carcinoma were analysed under light microscope. Histologic grade for each type of adenocarcinoma using the VACURG, Gleason grading system and Gleason's histologic scores were noted. Associated prostatic tissue changes like tumor invasion, PIN and other prostatic lesions were also analysed.

AgNOR staining method of Smith and Crocker was done for every case and mean AgNOR count calculated. In all specimens, 100 nuclei of each lesion were examined using a x100 oil immersion objective and a x10 ocular. Lesional nuclei were taken at random for the counting procedure. Careful focusing allowed the nucleolar organizer regions (NOR) to be visualized as black dots arranged both in clusters and clumps and as individual "satellites" within the cell nucleus. To assess the reproducibility of the counting procedure, all counts were repeated.

\subsection{Statistical analysis}

Results are presented as Mean \pm SD and range for quantitative data and number and percentages for qualitative data. Group wise comparisons were made either by student t-test/ Mann-Whitney test. P value of 0.05 or less was considered for statistical significant.

\section{Results}

\subsection{Prostatic lesions}

Out of 50 prostatic specimens received, 40 cases were benign lesions and prostatic malignancy was diagnosed in 10 cases. Incidence of benign lesions was $80 \%$ and malignant lesions $20 \%$ in this study.

\subsection{Nature of prostatic biopsies}

Among the benign lesions, TURP was done in 40 cases and core biopsy in 10 cases. Malignant lesions were diagnosed in 8 TURP and 2 core biopsy specimens. Hence, TURP constituted $80 \%$ and SPP in $20 \%$ of the total specimens.

\subsection{Age}

Among 50 benign lesions, majority of the benign cases recorded at age group of 59-68 years. The youngest case observed in 45 years and oldest was in 82 years. The mean age group $\pm \mathrm{SD}$ for benign lesions is $63.5 \pm 9.2$ years.

Among 10 malignant lesions, majority of the cases were in 71- 80 years of age. Youngest person was 52 years and oldest person was 80 years old in this category. The mean age group $\pm \mathrm{SD}$ for malignant lesions is $69.5 \pm 8.5$ years. When age groups of benign and malignant lesions were compared, $\mathrm{P}=0.09$, which is not significant.

\subsection{Clinical features}

The most common clinical symptom of benign lesions was frequency in 35 patients, followed by hesitancy and nocturia in 33 and 30 patients respectively. Malignant lesions had common symptoms of hesitancy in 8 patients followed by nocturia and frequency in 6 and 5 patients respectively. However when both lesions were compared, no significant difference recorded between benign and malignant lesions.

\subsection{Mean AgNOR count in benign and malignant lesions}

Mann-Whitney test used to $\mathrm{i}$ the statistical differences between AgNOR counts of benign and malignant lesions with $\mathrm{t}=53.9, \mathrm{Z}=7.23$ and $\mathrm{P}<0.001$ which was considered highly significant.

\subsection{Total serum PSA levels}

Serum PSA levels above $10 \mathrm{ng} / \mathrm{ml}$ were observed in 10 benign specimens and 5 malignant specimens. However when both PSA values were compared, $\mathrm{P}=0.02$ which was highly significant. 
Table 1: Clinical presentations of prostatic lesions

\begin{tabular}{lccc}
\hline Symptom & $\begin{array}{c}\text { Benign } \\
\text { lesions } \\
(\mathbf{n = 4 0})\end{array}$ & $\begin{array}{c}\text { Malignant } \\
\text { lesions } \\
(\mathbf{n = 1 0})\end{array}$ & $\begin{array}{c}\text { Total } \\
(\mathbf{n = 5 0})\end{array}$ \\
Frequency & 35 & 5 & 40 \\
Nocturia & 30 & 6 & 36 \\
Urgency & 25 & 2 & 27 \\
Hesitancy & 33 & 8 & 41 \\
Poor stream & 22 & 2 & 24 \\
Dribbling & 15 & 4 & 19 \\
Retention & 32 & 7 & 39 \\
Hematuria & 1 & 5 & 6 \\
Fever \& Chills & 2 & 0 & 2 \\
Intermittent & 5 & 1 & 6 \\
Stream & & & \\
\hline
\end{tabular}

$\mathrm{P}<0.05$, significant.

Table 2: AgNOR score

\begin{tabular}{lcc} 
& Range & Mean AgNOR \pm SD \\
Benign lesions & 1.22 to 2.3 & $1.60 \pm 0.2$ \\
Malignant lesions & 4.52 to 7.4 & $5.49 \pm 0.7$ \\
\hline
\end{tabular}

Table 3: Serum PSA levels in prostatic lesions

$\begin{array}{cccc}\begin{array}{c}\text { PSA values } \\ (\mathbf{n g} / \mathbf{m l})\end{array} & \begin{array}{c}\text { Benign } \\ \text { lesions }\end{array} & \begin{array}{c}\text { Malignant } \\ \text { lesions }\end{array} & \text { Total } \\ 0-4.0 & 18 & 4 & 22 \\ 4.1-10.0 & 12 & 1 & 13 \\ 10.1-20.0 & 6 & 1 & 7 \\ >20+ & 4 & 4 & 8 \\ \text { Total } & 40 & 10 & 50\end{array}$

\subsection{Observation}

Out of 50 cases, 35 were BPH, 5 specimens belongs to nonspecific granulomatous prostatitis, 8 belongs to prostatic adenocarcinoma, 1 case with urothelial carcinoma and 1 case with both prostatic adenocarcinoma and urothelial carcinoma.

Foci of low grade PIN identified in 8 specimens. All the low grade PIN foci was associated with BPH. High grade PIN identified in 9 specimen. Out of these 2 HGPIN foci were seen in BPH and 7 were observed associated with adenocarcinoma. Miscellaneous features like cystic atrophy, chronic non-specific prostatitis, basal cell hyperplasia, stromal nodule, and transitional cell metaplasia were observed associated with these lesions.

\section{Inflammatory lesions}

In this study, 5 cases with nonspecific granulomatous prostatitis were recorded. Chronic non-specific prostatitis formed majority among inflammatory lesions and predominantly it was seen in BPH cases and also in few cases of prostatic adenocarcinoma. Destruction of glandular lining noted at places with necrotic debri and inflammatory cells in lumen. The cases of non-specific granulomatous prostatitis shows the dense infiltrate of lymphocytes, plasma cells, foamy histiocytes, and few multinucleated giant cells. Foci of glands showed destruction of epithelial lining.

\section{Benign prostatic hyperplasia}

There was totally 35 cases of BPH. All BPH cases were in age group of 46-78 yrs. The peak incidence was observed in 60-69 yrs age group. The mean age of BPH belongs to 65.5 yrs. Sections consisted of proliferation of both glandular and stromal components. The glands were variable in size, at places showed cystic dilation and contained an inspissated secretion called corpora amylacea. The epithelium showed papillary in foldings. The outer basal cell layer composed of flattened and cuboidal cells placed on an intact basement membrane.

\subsection{Basal cell hyperplasia}

Basal cell hyperplasia identified in 3 cases. There were two patterns of basal cell hyperplasia observed.Stromal nodule shows the spindle cell proliferation in a hyalinized stroma with scattered thick walled blood vessels.

Foci of transitional cell metaplasia was observed in 1 case of BPH and was characterized by the presence of a stratified epithelium composed of oval to spindle cells perpendicularly oriented to the lumina, and having scanty pale eosinophilic to clear cytoplasm. The nuclei were elongated, vesicular with inconspicuous nucleoli.

\section{Premalignant lesions of the prostate}

\section{Prostatic intraepithelial neoplasia}

In our study, low grade PIN observed in 8 cases. All of these were associated with BPH. High grade PIN observed in 9 cases. Out of these 7 cases were associated with adenocarcinoma and 2 cases were seen in BPH. High grade PIN shows the high association with adenocarcinoma.

Low grade PIN showing the crowding and stratification of glandular secretory epithelium.

High grade PIN consisted of crowding and stratification of glandular secretory epithelium. The nuclei were enlarged with variation in size and shape and the nucleoli were prominent. The basal cells were intact but few cases showed discontinuity.

\subsection{Microscopic patterns of HGPIN}

There was four patterns identified in HGPIN, usually with multiple patterns in each case.

Tufting Pattern was observed in 2 specimen. Microscopy shows the neoplastic cells grow towards the lumen, forming wave- or mound-like structures.

Flat Pattern comprised 4 of out of 9 cases. Microscopy consisted of the glands lined with one or two layers of 
atypical cells without significant architectural abnormality.

Micropapillary pattern composed of glands lined by atypical secretory epithelial cells arranged in micropapillary structures, lacking fibrovascular cores was identified in 2 out of 9 cases of HGPIN. Cribriform Pattern showed glands with epithelium forming cribriform pattern was identified in 1 out of 9 cases.

In this present study the commonest pattern identified was tufting type and the next common pattern was flat type.

\section{Malignant lesions of prostate}

In our study, there were 8 cases of Adenocarcinoma, 1 case of Urothelial carcinoma and 1 case of Urothelial carcinoma were recorded. In malignant lesions, the peak incidence was observed in $9^{\text {th }}$ decade. All the cases of adenocarcinoma showed architectural disturbance, stromal invasion and nuclear anaplasia in the form of variable size and shape, hyperchromatic with prominent nucleoli.

Microscopic grading system followed in this study was Gleason's microscopic grading system of prostatic carcinoma.

\subsection{Gleason's Grading system}

The gleason score of 5 constituted in 1 case, score 7 in 1 case, score 8 in 2 cases, score 9 in 3 cases and score 10 in 1 case respectively. The majority of patients diagnosed as conventional adenocarcinoma had graded as score 9 (3 cases) followed by the score 8 ( 2 cases 8 ).

\section{Urothelialcarcinoma}

There was 2 cases of urothelial carcinoma found in this study and both were in $9^{\text {th }}$ decade of age. Case had both adenocarcinoma and urothelial carcinoma. Specimens were taken from the patients by TURP method. Microscopy shows the infiltrating sheets and solid nests of neoplastic transitional epithelial cells with moderate to scanty cytoplasm with pleomorphic and hyper chromatic nuclei having prominent nucleoli. The patient with urothelial carcinoma showed invasion into the bladder.

\section{Discussion}

The incidence of prostatic lesions increases with the advancing age, prostatic cancer is the second most common cancer among males. Diagnosis is usually done by microscopic study of transrectal core biopsy and TURP is the commonly performed surgery.

Among the inflammatory lesions, chronic prostatitis observed in majority cases and was highly associated with BPH. Non-specific granulomatous prostatitis observed in $4.6 \%$ ( 3 cases). Out of 50 prostatic specimens, 40 cases were benign lesions and prostatic malignancy was diagnosed in 10 cases. Incidence of benign lesions was $80 \%$ and

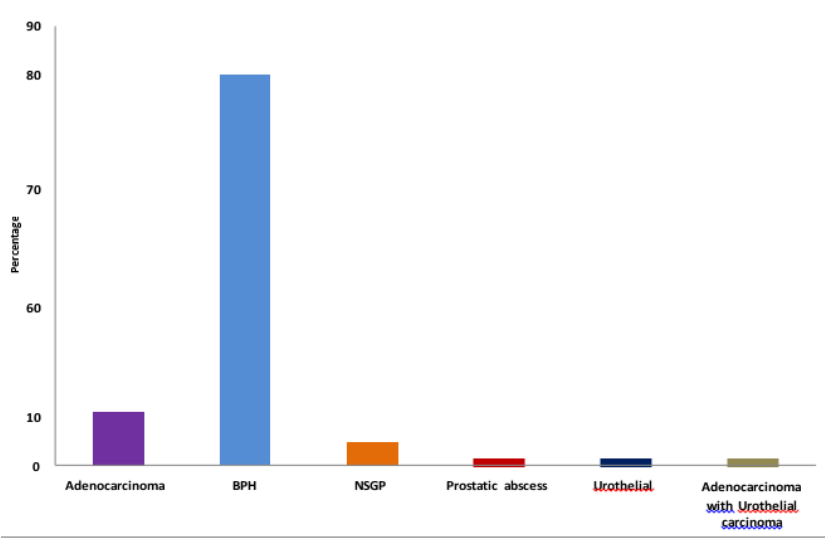

Fig. 1: Distribution of cases according to histopathological Diagnosis $(\mathrm{N}=50)$

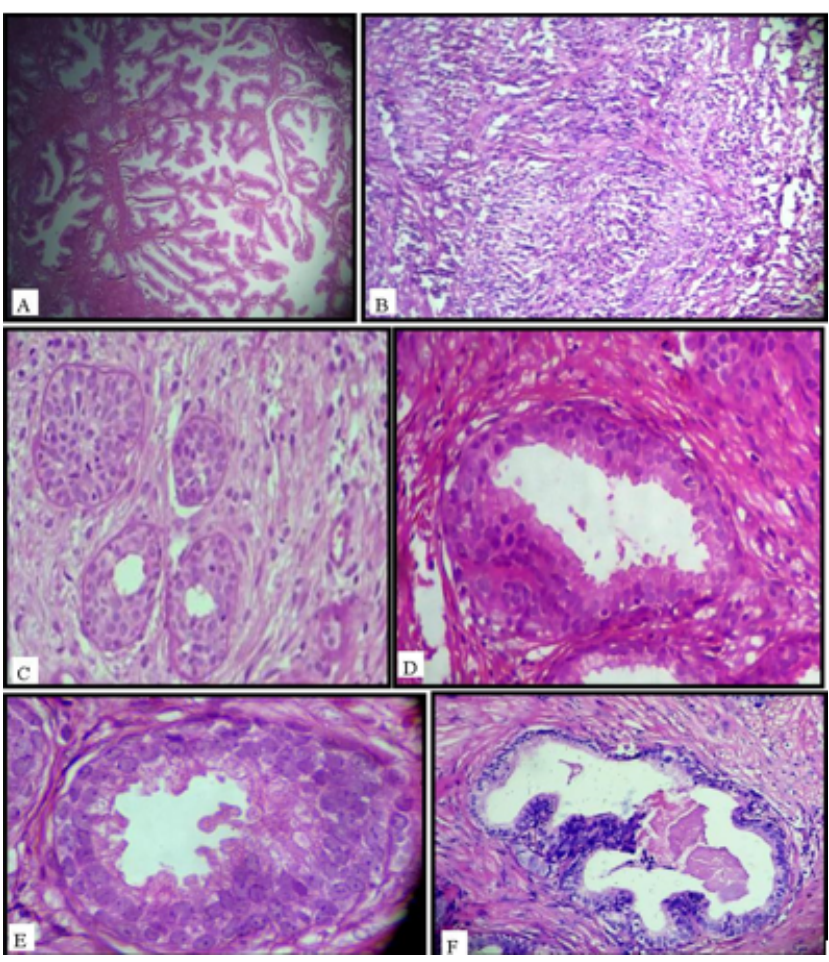

Fig. 2: A: Photomicrograph of Benign prostatic leison (10x), B: Photomicrograph of Chronic Prostatitis (10x). C: figure of Basal cell hyperplasia (10x), D: Photomicrograph of Low grade PIN (40x). E: Figure of High grade PIN-Tufting pattern.(40x). F: Photomicrograph of High grade Photomicrograph of Benign prostatic hyperplasiaN- Micropapillary pattern (40x). 


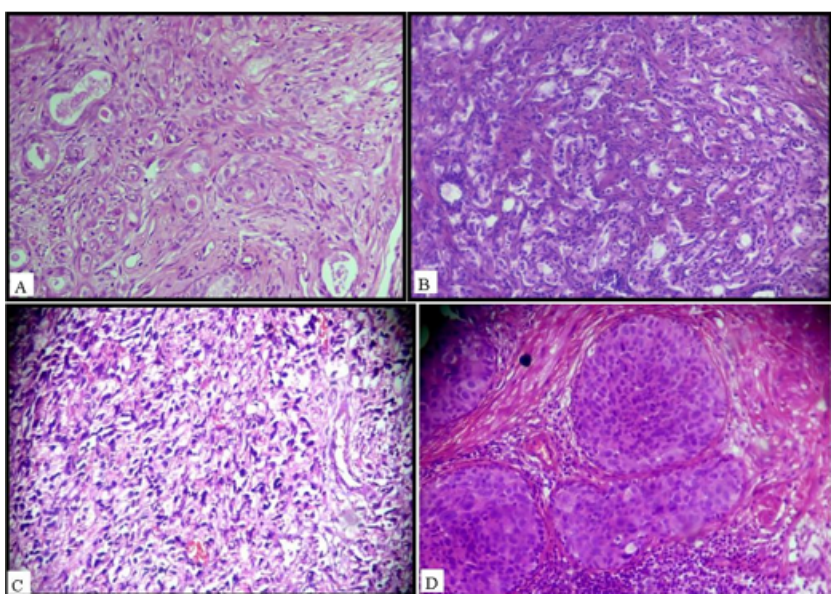

Fig. 3: A: Photomicrograph of Adenocarcinoma-Gleason's grade 2 (10x). B: Photomicrograph of Adenocarcinoma- Gleason's grade 3(10x). C: Photomicrograph of Adenocarcinoma-Gleason's grade 5(10x). D: Photomicrograph of Invasive Urothelial carcinoma $(10 \mathrm{x})$.

malignant lesions $20 \%$ in this study.

There were totally 35 cases of BPH. All these BPH cases were in 46-78 yrs age group. The peak incidence observed in of 60-69 yrs age group. The mean age of BPH in our study belongs to $65.5 \mathrm{yrs}$.

TURP constituted $80 \%$ and SPP in $20 \%$ of the total specimens. The mean age group $\pm \mathrm{SD}$ for benign lesions is $63.5 \pm 9.2$ years. In the benign proliferative lesions, BPH was seen in the majority of patients. Among 10 malignant lesions, the mean age group $\pm \mathrm{SD}$ for malignant lesions is $69.5 \pm 8.5$ years. Xie et al. ${ }^{5}$ found in their study that the percentage of carcinoma prostate was $84.2 \%$ over 65 years of age.

The most common clinical symptom of benign lesions was frequency in 35 patients, followed by hesitancy and nocturia in 33 and 30 patients respectively. Malignant lesions had common symptoms of hesitancy in 8 patients followed by nocturia and frequency in 6 and 5 patients respectively.

The present study showed 8 cases of LGPIN associated with BPH. Rekhi et al. ${ }^{6}$ found LGPIN in $18.6 \%$ cases of $\mathrm{BPH}$ and $5.8 \%$ of cases of adenocarcinoma.

In the present study HGPIN was observed in 9 cases, 2 HGPIN foci were seen in BPH and 7 were seen associated with adenocarcinoma. Malignant lesions, the peak incidence was seen in $9^{\text {th }}$ decade. The mean age of malignant cases was $75.5 \mathrm{yrs}$.

In out study, there were four microscopic patterns identified in HGPIN usually with multiple patterns in each case i.e. tufting, flat, micropapillary and cribriform patterns. The commonest pattern identified was tufting type followed by flat type.

Bostwick et al. ${ }^{7}$ in their study observed the percentage of tufting, flat, micropapillary and cribriform patterns $87 \%$,
$28 \%, 85 \%$ and $32 \%$ respectively. The commonest pattern was tufting type followed by micropapillarytype.

Out of $35 \mathrm{BPH}, 5$ were non-specific granulomatous prostatitis, 8 were prostatic adenocarcinoma, 1 case was urothelial carcinoma and 1 case had both prostatic adenocarcinoma and urothelial carcinoma.

Basal cell hyperplasia was identified in 3 cases out of 50 cases. Foci of transitional cell metaplasia was seen in 1 case of BPH. There were 2 cases of urothelial carcinoma found in this study and both were in $9^{t h}$ decade of age. Greene et al. ${ }^{8}$ in their study found the age distribution of urothelial carcinoma between $45-90$ years.

Serum PSA values above $10 \mathrm{ng} / \mathrm{ml}$ were seen in 10 benign cases and 5 malignant cases. PSA value in lesions with HGPIN is $11-22 \mathrm{ng} / \mathrm{ml}$. However when both PSA values were compared, $\mathrm{P}=0.02$ which was highly significant. Any associated prostatitis or malignancy may be responsible for elevation. The cause for the disparity is probably the late identification in symptomatic phase by malignancies in Indian scenario at which the cancer has already spread locally to increased volume of cancer tissue which in turn leads to increased PSA.

In our study, the gleason score 5, 7, 8, 9, 10 constituted 1 case, 1 case, 2 cases, 3 and 1 case respectively. Majority patients diagnosed as conventional adenocarcinoma had graded as score 9 ( 3 cases) followed by score 8 ( 2 cases 8 ). Gleason score of 8 and 9 was most commonly seen pattern, which is concordance with studies conducted by Deshmukh et al, Chauhan et al, Josephine et al. and Sujatha et al. ${ }^{9-12}$

\section{Conclusion}

The incidence of prostatic lesions is $20 \%$ among the various specimens received in the department during the period of one year. BPH was the highest observation within the prostate. Chronic nonspecific prostatitis is commonest inflammatory condition. Granulomatous prostatitis is rarely encountered. Conventional adenocarcinoma is common type of prostatic carcinoma. Gleason's score of 8-10 is common score in adenocarcinoma of prostate. High grade PIN has high degree of association with prostatic carcinoma. This reflects greater possibility of high grade PIN as a precursor lesion.

\section{Source of Funding}

None.

\section{Conflict of Interest}

None.

\section{References}

1. Bostwick DG, John N. Urologic Surgical Pathology . St Louis, United States: Elsevier - Health Sciences Division; 2018. 
2. Dabbs DJ. Diagnostic Immunohistochemistry. Churchill Livingstone; 2006.

3. Lf G, Farrow GM, Jm R, Fm T. John D Bancroft, Marilyn Gamble Theory and practice of histological techniques. J Urol. 1979;121:3035 .

4. Young RH, Srigley JR, Arvin MB, Ulbright TM, Antonio L. Tumors of the Prostate, Seminal Vesicles, Male Urethra and Penis: 28 (Atlas of Tumor Pathology (AFIP); 2000

5. Xie LP, Qin J, Zheng XY, Shen HF, Chen ZD, Cai SL, et al. Age and pathological features of 481 prostate cancer patients. Zhonghua nan ke xue $=$. Nat $J$ Androl. 2005;11(6):428-30.

6. Rekhi B, Jaswal TS, Arora B. Premalignant lesions of prostate and their association with nodular hyperplasia and carcinoma prostate. Indian J Cancer. 2004;41(2):60.

7. Bostwick DG, Algaba F, Amin MB, Ayala A, Eble J, Goldstein N, et al. Consensus statement on terminology: Recommendation to use atypical adenomatous hyperplasia in place of adenosis of the prostate. Human Pathol. 1994;25(8):840.

8. Greene LF, O'Dea MJ, Dockerty MB. Primary Transitional Cell Carcinoma of the Prostate. J Urol. 1976;116:761-3.

9. Deshmukh BD, Ramteerthakar NA, Sulhyan KR. Histopathological study of lesions of prostate - A five year study. Int J Health Sci Res.
2014;4(1):1-9.

10. Chauhan SC, Sarvaiya NA. Study of clinicomorphologic spectrum of prostatic lesions and correlation with prostate specific antigen levels in a tertiary care center. Indian J Pathol Oncol. 2017;4(2):328-32.

11. J A. Clinicopathological study of prostatic biopsies. J Clin Diagn Res. 2014;8(9):4-6.

12. Jaishree T, Sujatha R, and YAM. Analysis of spectrum of Prostate lesions in correlation with Serum Prostate specific antigen levels A clinicopathological study in a tertiary care centre. J Diagn Pathol Oncol. 2019;4(3):175-9.

\section{Author biography}

Tadepalli Krishna Associate professor

Cite this article: Krishna T. A clinico-histopathological spectrum of Prostatic lesions at a tertiary care teaching institute. IP J Diagn

Pathol Oncol 2020;5(3):300-305. 